\title{
Weicon setzt auf klebtechnische Ausbildung
}

Der Kleb- und Dichtstoffhersteller Weicon nimmt bereits seit 2004 die vom Fraunhofer-Institut für Fertigungstechnik und Angewandte Materialforschung (IFAM) in Bremen angebotenen Aus- und Weiterbildungsangebote in Anspruch.

$\mathrm{M}$ ittlerweile haben 60 Mitarbeiterinnen und Mitarbeiter einen Lehrgang in Bremen absolviert und können sich als Klebpraktiker, Klebfachkraft oder als Klebfachingenieur bezeichnen. Damit ist bei Weicon inzwischen die Hälfte der gesamten Belegschaft zu Fachkräften weitergebildet worden.

Diese Spezialisten im Bereich der Klebtechnik können ihr Wissen in vielen Bereichen wie der Beratung von Kunden oder bei der Forschung und Entwicklung neuer Produkte einsetzen.

Laut Aussage des IFAM in Bremen beschäftigt Weicon im Bereich der Klebtechnik im Verhältnis zur gesamten Mitarbeiterzahl die meisten Fachkräfte. „Der Erfolg der Schulungsmaßnahmen wird nicht nur durch die Zertifikate des IFAM belegt, sondern auch durch das Feedback zufriedener Kunden, die sich bei Fragen stets an unsere Fachkräfte wenden können und bestens betreut werden”, so Ralph Weidling, Geschäftsführer des Münsteraner Familienunternehmens. „Durch die Weiterbildungen beugen wir aktiv dem drohenden Fachkräftemangel vor und sorgen für die gesicherte Zukunft unseres Unternehmens“, so Weidling weiter.

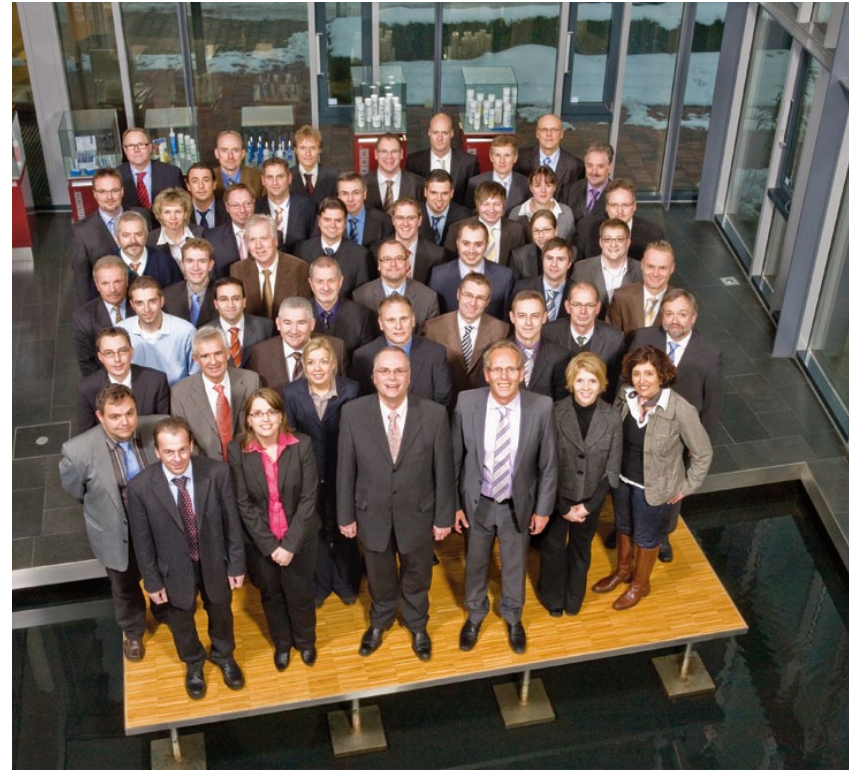

Weicon hat bereits die Hälfte der gesamten Belegschaft zu klebtechnischen Fachkräften weiterbilden lassen.

\section{Delo erhält Trainee-Gütesiegel}

Delo ist jetzt mit einem Trainee-Gütesiegel ausgezeichnet worden. Initiatoren der Auszeichnung sind die Absolventa GmbH, Jobnet sowie die Süddeutsche Zeitung.

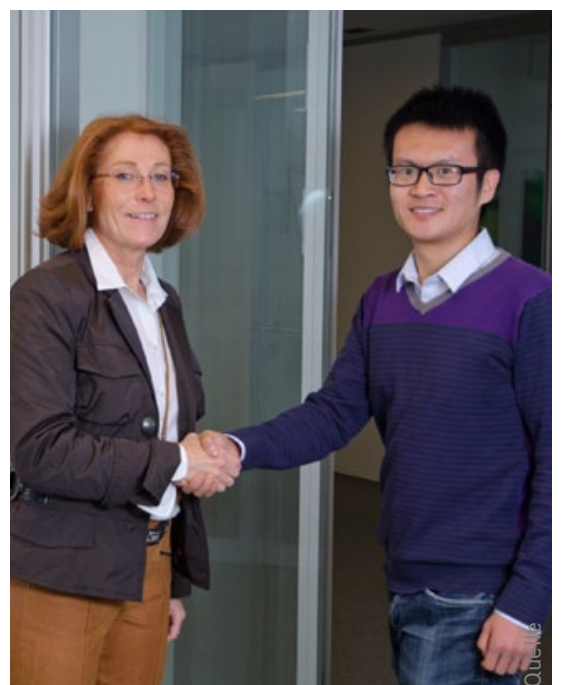

Sabine Herold, geschäftsführende Delo-Gesellschafterin, neben Mengbo Zhou, der ein Jahr lang als Trainee bei Delo in Windach arbeiten wird, bevor er als Applikationsingenieur die Shanghai-Niederlassung verstärkt $\bigvee \begin{aligned} & \text { oraussetzung für das Gütesiegel } \\ & \text { waren verschiedene Kriterien, die }\end{aligned}$ es zu erfüllen galt. „Unsere Trainees übernehmen von Anfang an verantwortungsvolle Aufgaben“, so Sabine Herold, geschäftsführende Delo-Gesellschafterin. Außerdem lege das Unternehmen Wert auf umfangreiche Schulungen sowie ein faires Gehalt. Delo bietet spezielle Traineeprogramme für Ingenieure an, die zunächst sämtliche Abteilungen des Unternehmens durchlaufen, Produktschulungen erhalten und selbst Klebstoffversuche durchführen dürfen. Danach werden sie in verschiedenen Unternehmensbereichen eingesetzt, z.B. im Vertrieb oder Produktmanagement.

Die Karrierewege bei Delo sind vielfältig: „Einer unserer Mitarbeiter hat als Trainee begonnen, anschlie- ßend unsere Shanghai-Niederlassung aufgebaut und leitet jetzt unsere Repräsentanz in Boston, USA“, so Sabine Herold. Ein anderer Trainee habe 2005 direkt nach seinem Chemie-Ingenieurs-Studium an der TU München begonnen, sei anschließend im internationalen Vertrieb für UK und Spanien zuständig gewesen und leite heute den Vertrieb Europa. „Ich selbst habe als Anwendungsingenieurin bei Delo gestartet und bin dann über Marketing und Vertrieb in die Geschäftsführung gekommen.“

Erst Anfang November 2012 startete ein weiterer Trainee: Mengbo Zhou, chinesischer Absolvent der Universität Saarbrücken, wird in Windach ein Jahr als Trainee arbeiten, bevor er als Applikationsingenieur die Shanghai-Niederlassung verstärkt. 